\title{
Use of ß-blockers and mortality following ovarian cancer diagnosis: a population-based cohort study
}

Sigrun A Johannesdottir ${ }^{1,2^{*}}$, Morten Schmidt ${ }^{1,2}$, Gary Phillips ${ }^{3}$, Ronald Glaser ${ }^{4,5,6}$, Eric V Yang ${ }^{5,6}$, Michael Blumenfeld ${ }^{4}$ and Stanley Lemeshow ${ }^{1,2}$

\begin{abstract}
Background: Experimental data suggest that catecholamine hormones are involved in stimulating the aggressiveness of ovarian cancer, but few population-based studies have examined this association. We therefore conducted a population-based cohort study to examine whether ß-blockers affect mortality following ovarian cancer diagnosis.
\end{abstract}

Methods: We used the Danish Cancer Registry to identify all patients diagnosed with ovarian cancer in northern Denmark between 1999 and 2010 ( $n=6,626)$. Data on medication use, comorbidity, and survival were obtained from medical databases. According to the last redeemed prescription before diagnosis, ß-blocker use was categorized as current (within $\leq 90$ days), previous (>90 days) or never. We used Cox proportional hazards regression to calculate hazard ratios (HRs) for all-cause mortality with 95\% confidence intervals (Cls) adjusting for confounding factors.

Results: Among the ovarian cancer patients, 373 (5.6\%) were current, 87 (1.3\%) previous, and 6,166 (93.1\%) were nonusers of $ß$-blockers. Median duration of use was 19.0 months among current users and 43.0 months among previous users. Median follow-up was 2.55 years (IQR: 0.81-9.23). Nonusers and current users of ß-blockers had similar comorbidity burden whereas previous users had moderate comorbidity more frequently. Compared with nonusers, the adjusted HR was 1.17 (95\% Cl: 1.02-1.34) for current users and 1.18 (95\% Cl: 0.90-1.55) for previous users. Secondary analyses stratifying by cancer stage and duration of $ß$-blocker use supported the overall results.

Conclusions: We found no evidence that ß-blocker use was associated with decreased mortality following ovarian cancer diagnosis.

\section{Background}

Inhibiting the sympathetic actions of catecholamine hormones (i.e., epinephrine and norepinephrine), ß-blockers are used for various indications, particularly cardiac arrhythmias, cardioprotection after myocardial infarction, hypertension, migraine, and tremor [1]. These diverse indications reflect the abundance of $\beta$-adrenoceptors in the body. Experimental evidence shows that malignant cell lines from, e.g., ovarian cancer and malignant melanoma also express $\beta$-adrenoceptors and that catecholamine stress

\footnotetext{
*Correspondence: saj@dce.au.dk

'Division of Biostatistics, College of Public Health, The Ohio State University, Columbus, OH, USA

2Department of Clinical Epidemiology, Aarhus University Hospital, Aarhus N, Denmark

Full list of author information is available at the end of the article
}

hormones may affect carcinogenesis through these receptors [2-8].

Previous research on the association between $ß$-blocker use and mortality following malignant melanoma, have shown consistent results between the protective effects observed ex-vivo and in a population-based setting [7-9]. However, data on the effect of ß-blockers on mortality following ovarian cancer in a population-based setting are sparse [10]. We therefore conducted a population-based cohort study to examine whether use of $ß$-blockers are associated with mortality in patients with ovarian cancer.

\section{Methods}

\section{Setting}

The Danish National Health Service guarantees the entire Danish population universal tax-supported health care

\section{Biomed Central}


including access to general practitioners and hospitals as well as partial reimbursement of various drugs including ß-blockers [11-13]. All individuals residing in Denmark at any point in time since 1968 are assigned a unique central personal registration (CPR) number, which is used to record health-related services in various nationwide registries and allows accurate and unambiguous individual-level linkage of all registries [13].

We conducted this population-based cohort study in northern Denmark (population 1.7 million, $\approx 30 \%$ of the Danish population). This region encompasses the former North Jutland County, Aarhus County, Viborg County and Ringkøbing County for which complete computerized prescription records are available through the Aarhus University Prescription Database since 1 January 1998 [12]. By starting the study period on 1 January 1999, we ensured a minimum of one year of prescription history for all participants in the study.

\section{Study cohort}

The Danish Cancer Registry (DCR) has recorded information on all incident malignant neoplasms in Denmark since 1943 [14]. Tumors are classified according to the $7^{\text {th }}$ revision of the International Classification of Diseases (ICD-7) from 1943 through 2003 and according to the $10^{\text {th }}$ revision thereafter [14].

We used the DCR to identify all women with a firsttime diagnosis of ovarian cancer from 1 January 1999 to 31 December 2010. We also included information on stage at diagnosis according to the Summary Staging classification with the TNM grouping translated as follows: localized (TNM: T1-4, N0, M0), regional (TNM: Tx, N13, M0), distant (TNM: Tx, N1-3, M1), or unknown/ missing. We included only women aged 20 years or more at time of diagnosis.

\section{ß-blocker use}

Using the Aarhus University Prescription Database [12], we identified all prescriptions for ß-blockers redeemed by study subjects before their diagnosis date. For each prescription dispensed, the patient's CPR number, type and amount of drug prescribed according to the Anatomical Therapeutic Chemical (ATC) classification system, and date of dispensation, are recorded in the electronic accounting system at the pharmacy and subsequently transferred to the database [12].

We defined three exposure categories: (1) current users were those redeeming at least one prescription within 90 days of ovarian cancer diagnosis, (2) previous users were those redeeming their last prescription in the interval 91 to 365 days prior to ovarian cancer diagnosis, and (3) nonusers were those with no prescription records of $\beta$ blocker use within 365 days of ovarian cancer diagnosis. We assessed exposure status prior to diagnosis to avoid introducing immortal time bias, i.e., that users would appear to survive longer because a user by definition had to survive to become a user [15]. We chose to assess exposure prior to diagnosis to mimic an intention-to-treat method [9] and because we hypothesized that ß-blockers exert an inhibitory effect early in ovarian cancer progression. In Denmark, most prescriptions cover 30 to 90 days. To assure that we captured most current users, we therefore chose 90 days as the exposure window.

\section{Other patient characterstics}

We used the Aarhus University Prescription Database to obtain information on comedication use of the following agents between establishment of computerized prescription registries in each county and the date of ovarian cancer diagnosis: angiotensin-converting enzyme (ACE) inhibitors, calcium channel blockers, diuretics, statins, antidepressant drugs, antipsychotics, anxiolytic drugs, and hormone replacement therapy. Over-the-counter use of aspirin and low-dose ibuprofen is not recorded in the database [12]. However, regular users are typically registered in the prescription database because the cost is automatically partly refunded when a physician prescribes the drug [12].

The Danish National Registry of Patients (DNRP) has recorded all inpatient admissions to non-psychiatric hospitals since 1977, and all outpatient and emergency admissions since 1995 [16]. Each record includes information on dates of admission and discharge, diagnosis codes and associated surgical procedures [16]. Diagnoses are classified according to ICD-8 through 1993 and the ICD-10 revision thereafter [16]. Surgical procedures are recorded using a Danish version of the Nordic Medico-Statistical Committee (NOMESCO) Classification of Surgical Procedures [16]. We used the DNPR to retrieve information on any diagnosis of obesity, atrial fibrillation/flutter, angina pectoris, myocardial infarction, congestive heart failure, esophageal varices, tremor, anxiety, thyrotoxicosis, chronic obstructive pulmonary disease (COPD), migraines, stroke, chronic kidney disease, or a history of hysterectomy or tubal sterilization prior to ovarian cancer diagnosis. We also measured the comorbidity burden using the Charlson Comorbidity Index (CCI) categorized as low (score 0), moderate (score 1-2), or high (score $\geq 3$ ). The $\mathrm{CCI}$ is an extensively studied and validated instrument used to predict the risk of death from comorbid diseases, by covering and weighing 19 major chronic disease categories based on the relative risk of dying [17-21].

All ICD and ATC codes used in the study are provided in the Additional file 1: ATC and ICD codes.

\section{Mortality}

We started follow-up on the date of diagnosis and continued until death, emigration, or end of follow-up (31 December 2010) whichever came first. We identified 
all-cause mortality using the Danish Civil Registration System. The Danish Civil Registration System was established on 1 April 1968 and includes daily updates on changes in migration and vital status for the entire Danish population [13].

\section{Statistical analysis}

Initially, we computed the frequency and proportion of covariates, number of deaths, and amount of accumulated person-time within categories of ß-blocker use. Using time since diagnosis as the time scale, we then used Cox proportional hazard regression to estimate hazard ratios (HRs) with 95\% confidence intervals (CIs) associating ß-blocker use with mortality both overall and according to stage at diagnosis. We used a risk-factor modeling approach to fit a multivariable model. Age group (20-40, 41-60, 61-80, and $>80$ years) and CCI level were variables of interest a priori and therefore included in the model from the start. Adding the remaining variables to the model one at the time resulted in a less than $10 \%$ change in the $\mathrm{HR}$ associated with ß-blocker use. However, including prior use of diuretics, aspirin, and statins, collectively in the model demonstrated substantial confounding $(>10 \%)$ and they were therefore included in the model. We then tested for statistically significant interactions between any of the covariates and $\beta$-blocker use. We assessed the assumption of proportional hazards by graphical examination of log-log plots and found it not to be violated.

We performed two secondary analyses according to duration of use (calculated as time between first and last prescription plus 90 days). First, we made a restriction to current users with at least one year of $ß$-blocker use and compared them to non-users. Second, we examined the association between $\beta$-blockers and mortality following ovarian cancer according to months of use among all user groups. To ensure at least five years of prescription history and thereby limit left censoring, this analysis was restricted to women diagnosed in 2003-2010. The method of fractional polynomials was used to confirm that months of use, was linear in the log-hazard function.

We performed all analyses using STATA $^{\circledR}$ software (version 11.0, STATA, College Station, TX). The study was approved by the Danish Data Surveillance Authority.

\section{Results}

\section{Patients characteristics}

Table 1 presents the characteristics of the 6,626 ovarian cancer patients included in the study. Median age at diagnosis was overall 65 years (between 63-66 years for all exposure groups). There were 6,166 (93.1\%) nonusers, 373 (5.6\%) current users, and 87 (1.3\%) previous users of $\beta$-blockers in the cohort (Table 1). The median duration of use was 19.0 months among current users and 43.0 months among previous users (Table 1).

Current and previous users of $ß$-blockers had more frequently localized stage at the time of diagnosis than nonusers. Stage was unknown/missing for $7.6 \%$ of nonusers, $9.9 \%$ of current users, and $11.5 \%$ of previous users. Nonusers and current users of ß-blockers had similar comorbidity burden whereas previous users had more frequently moderate comorbidity. Current and previous users of $ß$-blockers more frequently used the comedications identified.

\section{Mortality}

Overall the median follow-up time was 2.55 years (IQR: 0.81-9.23). Compared with nonusers, the adjusted HR was 1.17 (95\% CI: 1.02-1.34) for current users and 1.18 (95\% CI: 0.90-1.55) for previous users (Table 2). When examining the association according to stage at time of diagnosis, we found similar results (Table 2).

There were no statistically significant interactions between any of the covariates and ß-blocker use, except for age. To examine the clinical relevance, we therefore stratified the results by age group. Although rather imprecise, the estimates indicated a tendency towards higher HRs in older age groups (Table 1 in Additional file 2: Tables for secondary analyses and stratification by age). The imprecise estimates for the age group below 40 years, made this strata inconclusive.

The secondary analysis showed that the results for current users with $\geq 1$ year duration of use were not substantially different from the results including all current users (Table 2 in Additional file 2: Additional tables). There was no association between months of use entered as a linear variable and mortality following ovarian cancer (HR 1.01; 95\% CI: 1.00-1.01, Table 3 in Additional file 2: Additional tables).

\section{Discussion}

In this population-based cohort study, we found no evidence of an association between ß-blocker use and decreased mortality following a diagnosis of ovarian cancer.

Previous in vitro data on cancer cell lines suggest that ß-blockers exert an antitumor effect via direct action on ovarian cancer cells [2-4]. It has been shown that treatment with adrenergic agonists could upregulate the production of matrix metalloproteinase (MMP)-2, MMP-9, and VEGF in ovarian cancer cell lines resulting in increased invasive capability $[2,4]$. This effect was mediated through $\beta$-adrenoceptors and was blocked by treatment with the $ß$-antagonist propranolol $[2,4]$. Similar results have been found for other malignancies, e.g., malignant melanoma, multiple myeloma, and nasopharyngeal carcinoma [5-8,22-25]. Finally, it has been shown that 
Table 1 Ovarian cancer patient demographics by ß-blocker use

\begin{tabular}{|c|c|c|c|}
\hline & Nonusers & Current users & Previous users \\
\hline Number (\%) & $6,166(93.06)$ & $373(5.63)$ & $87(1.31)$ \\
\hline \multicolumn{4}{|l|}{ Age at diagnosis, years } \\
\hline Median age (IQR) & $65(56-75)$ & $66(56-74)$ & $63(55-73)$ \\
\hline $20-40$ & $213(3.45)$ & $13(3.49)$ & $1(1.15)$ \\
\hline $41-60$ & $1,982(32.14)$ & $118(31.64)$ & $32(36.78)$ \\
\hline $61-80$ & $3,188(51.70)$ & $197(52.82)$ & $42(48.28)$ \\
\hline$>80$ & $783(12.70)$ & $45(12.06)$ & $12(13.79)$ \\
\hline \multicolumn{4}{|l|}{ Stage ${ }^{b}$} \\
\hline Localized & $1,848(29.97)$ & $128(34.32)$ & $28(32.18)$ \\
\hline Regional & $1,931(31.32)$ & $97(26.01)$ & $16(18.39)$ \\
\hline Distant & $1,920(31.14)$ & $111(29.76)$ & $33(37.93)$ \\
\hline Unknown/missing & $467(7.57)$ & 37 (9.92) & $10(11.49)$ \\
\hline \multicolumn{4}{|l|}{ Comorbidity level ${ }^{c}$} \\
\hline Low & $4,127(66.93)$ & $251(67.29)$ & $58(66.67)$ \\
\hline Moderate & $1,512(24.52)$ & $88(23.59)$ & $26(29.89)$ \\
\hline High & $527(8.55)$ & 34 (9.12) & $3(3.45)$ \\
\hline \multicolumn{4}{|l|}{ Comorbidities } \\
\hline Hypertension & $681(11.04)$ & $45(12.06)$ & $12(13.79)$ \\
\hline Obesity & $71(1.15)$ & $2(0.54)$ & $0(0.00)$ \\
\hline Atrial fibrillation/flutter & $222(3.60)$ & $22(5.90)$ & $8(9.20)$ \\
\hline Angina pectoris & $257(4.17)$ & $9(2.41)$ & $0(0.00)$ \\
\hline Myocardial infarction & $160(2.59)$ & $10(2.68)$ & $2(2.30)$ \\
\hline Congestive heart failure & $183(2.97)$ & $12(3.22)$ & $4(4.60)$ \\
\hline Esophageal varices & $5(0.08)$ & $1(0.27)$ & $0(0.00)$ \\
\hline Tremor & $4(0.06)$ & $0(0.00)$ & $0(0.00)$ \\
\hline Anxiety & $41(0.66)$ & $1(0.27)$ & $1(1.15)$ \\
\hline Thyrotoxicosis & $182(2.95)$ & $16(4.29)$ & $5(5.75)$ \\
\hline COPD & $227(3.68)$ & $13(3.49)$ & $4(4.60)$ \\
\hline Migraines & $78(1.27)$ & $3(0.80)$ & $2(2.30)$ \\
\hline Stroke & $223(3.62)$ & $21(5.63)$ & $4(4.60)$ \\
\hline Chronic kidney disease & $81(1.31)$ & $5(1.34)$ & $3(3.45)$ \\
\hline Hysterectomy & $358(5.81)$ & $24(6.43)$ & $7(8.05)$ \\
\hline Tubal sterilization & $204(3.31)$ & $11(2.95)$ & $3(3.45)$ \\
\hline \multicolumn{4}{|l|}{ Comedication use } \\
\hline ACE-inhibitors & $351(5.69)$ & $104(27.88)$ & $30(34.48)$ \\
\hline ARBs & $198(3.21)$ & $60(16.09)$ & 16 (18.39) \\
\hline Calcium channel blockers & $407(6.60)$ & $112(30.03)$ & 31 (35.63) \\
\hline Diuretics & $1,276(20.69)$ & $234(62.73)$ & $67(77.01)$ \\
\hline Non-aspirin NSAIDs & $1,852(30.04)$ & $220(58.98)$ & $51(58.62)$ \\
\hline Aspirin & $525(8.51)$ & $157(42.09)$ & $40(45.98)$ \\
\hline Statins & $320(5.19)$ & $111(29.76)$ & $28(32.18)$ \\
\hline
\end{tabular}


Table 1 Ovarian cancer patient demographics by B-blocker use (Continued)

\begin{tabular}{lrrr}
\hline HRT & $1,165(18.89)$ & $126(33.78)$ & $33(37.93)$ \\
Antipsychotics & $336(5.45)$ & $41(10.99)$ & $8(9.20)$ \\
Anxiolytics & $285(4.62)$ & $22(5.90)$ & $7(8.05)$ \\
Antidepressants & $816(13.23)$ & $100(26.81)$ & $24(27.59)$ \\
\hline
\end{tabular}

ACE: angiotensin-converting enzyme; ARBs: angiotensin receptor blockers; NSAIDs: nonsteroidal anti-inflammatory drugs.

a Defined as time between first and last prescription plus 90 days (assumed to be the average length of prescription).

${ }^{b}$ Classified according to Summary Staging classification with the TNM grouping translated as localized (TNM: T1-4, N0, M0), regional (TNM: T1-4, N1-3, M0),

distant (TNM: T1-4, N1-3, M1), or unknown/missing.

${ }^{c}$ Computed using the Charlson Comorbidity Index (CCl) score categorized into low (0), medium (1-2), or high (3+).

presurgical ovarian cancer patients with low social stress have lower VEGF levels possibly linking stress, and hence $ß$-adrenergic agents, to tumor angiogenesis [3].

In a recent meta-analysis, the association between $B$ blocker use and cancer-related mortality was examined and no association was found (odds ratio $=0.93$; 95\% CI: $0.80-1.08$ ) [26]. However, this study examined only the association for cancer-related mortality overall [26]. To our knowledge, only one previous study has examined this association for ovarian cancer specifically [10]. Diaz et al. [10] examined the association between B-blockers and disease progression and survival among 248 patients with stage III or IV epithelial ovarian or primary peritoneal cancer who had undergone primary exploratory laparotomy followed by at least 6 cycles of platinumand taxane-based chemotherapy. They found an median survival of 56 months for ß-blocker users compared with 48 months for non-users [10]. However, the interpretabilty of the results is hampered for several reasons. First, the study included a selected patient population, which limits generalizability. Second, the improved survival seemed confined to the later part of follow-up (more than 2-3 years), which is associated with statistical uncertainty because only few patients survived that long. Finally, and most importantly, because authors' decided that to be considered as exposed a patient had to have used ß-blockers for at least 6 months, they also had to have survived at least 6 months, and therefore immortal time bias may entirely explain the increased survival among ß-blocker users $[10,15]$. By assessing exposure prior to start of follow-up, we circumvented this problem in our study, which did not support any effect on the mortality rate after ovarian cancer

Table 2 Mortality hazard ratio ${ }^{a}$ following ovarian cancer diagnosis associated with B-blocker use, overall and by cancer stage at diagnosis ${ }^{b}$

\begin{tabular}{|c|c|c|c|c|}
\hline & Number of deaths (\%) & Median years of follow-up & Crude HR $(95 \% \mathrm{Cl})$ & Adjusted HR $(95 \% \mathrm{CI})^{\mathrm{C}}$ \\
\hline \multicolumn{5}{|l|}{ Overall } \\
\hline Nonusers & $4,106(66.59)$ & 2.56 & 1 (reference) & 1 (reference) \\
\hline Current users & $245(65.68)$ & 2.65 & $1.04(0.91-1.18)$ & $1.17(1.02-1.34)$ \\
\hline Previous users & $55(63.22)$ & 2.14 & $1.03(0.79-1.35)$ & $1.18(0.90-1.55)$ \\
\hline \multicolumn{5}{|c|}{ Localized cancer } \\
\hline Nonusers & $722(39.07)$ & 10.58 & 1 (reference) & 1 (reference) \\
\hline Current users & $46(35.94)$ & 7.87 & $0.97(0.72-1.31)$ & $1.01(0.73-1.39)$ \\
\hline Previous users & $13(46.43)$ & 7.07 & $1.44(0.83-2.50)$ & $1.57(0.90-2.76)$ \\
\hline \multicolumn{5}{|c|}{ Regional metastasis } \\
\hline Nonusers & $1,409(72.97)$ & 2.54 & 1 (reference) & 1 (reference) \\
\hline Current users & $75(77.32)$ & 2.21 & $1.21(0.96-1.52)$ & $1.52(1.18-1.95)$ \\
\hline Previous users & $10(62.50)$ & 1.42 & $0.95(0.51-1.77)$ & $0.89(0.46-1.69)$ \\
\hline \multicolumn{5}{|c|}{ Distant metastasis } \\
\hline Nonusers & $1,602(83.44)$ & 1.25 & 1 (reference) & 1 (reference) \\
\hline Current users & $96(86.49)$ & 1.10 & $1.09(0.89-1.35)$ & $1.18(0.95-1.47)$ \\
\hline Previous users & $24(72.73)$ & 1.86 & $0.70(0.47-1.05)$ & $0.91(0.60-1.37)$ \\
\hline
\end{tabular}

Cl: Confidence interval.

a Obtained using Cox proportional hazards models.

${ }^{b}$ Classified according to Summary Staging classification with the TNM grouping translated as localized (TNM: T1-4, N0, M0), regional (TNM: T1-4, N1-3, M0), distant (TNM: T1-4, N1-3, M1), or unknown/missing.

c Adjusted for age (20-40, 41-60, 61-80, $\geq 80$ years), comorbidity level, prior use of diuretics (yes/no), year of diagnosis, aspirin (yes/no), and statins (yes/no). Comorbidity was computed using the Charlson Comorbidity Index score categorized into low (0), medium (1-2), or high (3+). 
diagnosis associated with prior ß-blocker use. However, we cannot rule out an increased or decreased mortality for individual agents or certain histological types of ovarian cancer. Furthermore, given the wide confidence intervals and the non-randomized design, we are unable to exclude a small protective effect on mortality following ovarian cancer diagnosis.

Several issues should be considered when interpreting our results. The study's population-based design within the setting of a tax supported universal healthcare system reduces selection biases. Also, we were able to link population-based registries with complete data on drug use, cancer diagnosis, outpatient visits, and hospitalizations $[12,14,19,27,28]$. For example, the positive predictive values of diagnoses in the Danish National Registry of Patients have previously been validated and found to exceed $98 \%$ for Charlson comorbidities overall [19].

Data in the prescription database are virtually complete [12]. Although we had to use prescription data as a proxy for actual ß-blocker use, we did not base drug exposure information on prescription filling, but on actual dispensing at pharmacies [12]. Furthermore, we believe that copayment requirements increased the likelihood of compliance. However, any misclassification of drug exposure would most likely have been nondifferential and thus could, in part, explain the null result.

We were only able to consider all-cause mortality. We did not have any biological explanation to support that ß-blocker use would increase mortality rates following ovarian cancer. Thus, multiple comparisons may explain the slightly increased point estimates observed in some exposure categories. These estimates may also have resulted from uncontrolled confounding, as it is plausible that $ß$-blocker users on average are unhealthier than nonusers. However, the universal provision of health care considerably reduces the likelihood of substantial confounding by social characteristics. Furthermore, we did adjust partly for lifestyle factors by taking a history of obesity, COPD and ischemic heart disease into account. Still, due to the nonrandomized design, we cannot exclude uncontrolled confounding.

\section{Conclusions}

In conclusion, the present population-based cohort study does not provide evidence of an association between $ß$-blocker use and decreased mortality following a diagnosis of ovarian cancer.

\section{Additional files}

Additional file 1: ATC and ICD codes. A table presenting all ATC and ICD codes used in the study.

Additional file 2: Additional tables. Three tables presenting the results for the secondary analyses and stratification by age.
Competing interests

The authors declare that they have no competing interest.

\section{Authors' contributions}

RG, EVY, GP, and SL conceived the study idea. All authors reviewed the literature and/or designed the study. SAJ, MS, GP, and SL analyzed the data. All authors interpreted the findings. SAJ and MS organized the writing and wrote the initial draft. All authors edited the manuscript and approved the final version.

\section{Acknowledgements}

The study received financial support from the OSU College of Public Health.

\section{Author details}

${ }^{1}$ Division of Biostatistics, College of Public Health, The Ohio State University, Columbus, OH, USA. ²Department of Clinical Epidemiology, Aarhus University Hospital, Aarhus N, Denmark. ${ }^{3}$ The Ohio State University, Center for Biostatistics, Columbus, OH, USA. ${ }^{4}$ The Ohio State University Medical Center, Columbus, OH, USA. ${ }^{5}$ Institute for Behavioral Medicine Research, The Ohio State University, Columbus, OH, USA. ${ }^{6}$ Comprehensive Cancer Center, The Ohio State University Medical Center, Columbus, OH, USA.

Received: 26 October 2012 Accepted: 20 February 2013

Published: 22 February 2013

\section{References}

1. Ong HT: Beta blockers in hypertension and cardiovascular disease. BMJ 2007, 334:946-949.

2. Lutgendorf SK, Cole S, Costanzo E, Bradley S, Coffin J, Jabbari S, Rainwater K, Ritchie JM, Yang M, Sood AK: Stress-related mediators stimulate vascular endothelial growth factor secretion by two ovarian cancer cell lines. Clin Cancer Res 2003, 9(9):4514-4521.

3. Lutgendorf SK, Johnsen EL, Cooper B, Anderson B, Sorosky Jl, Buller RE, Sood AK: Vascular endothelial growth factor and social support in patients with ovarian carcinoma. Cancer 2002, 95:808-815.

4. Sood AK, Bhatty R, Kamat AA, Landen CN, Han L, Thaker PH, Li Y, Gershenson DM, Lutgendorf S, Cole SW: Stress hormone-mediated invasion of ovarian cancer cells. Clin Cancer Res 2006, 12:369-375.

5. Roy R, Zhang B, Moses MA: Making the cut: protease-mediated regulation of angiogenesis. Exp Cell Res 2006, 312:608-622.

6. Tammela T, Enholm B, Alitalo K, Paavonen K: The biology of vascular endothelial growth factors. Cardiovasc Res 2005, 65:550-563.

7. Tas F, Oguz H, Argon A, Duranyildiz D, Camlica H, Yasasever V, Topuz E: The value of serum levels of IL-6, TNF-alpha, and erythropoietin in metastatic malignant melanoma: serum IL- 6 level is a valuable prognostic factor at least as serum LDH in advanced melanoma. Med Oncol 2005, 22:241-246.

8. Ugurel S, Rappl G, Tilgen W, Reinhold U: Increased serum concentration of angiogenic factors in malignant melanoma patients correlates with tumor progression and survival. J Clin Oncol 2001, 19:577-583.

9. Lemeshow S, Sorensen HT, Phillips G, Yang EV, Antonsen S, Riis AH, Lesinski GB, Jackson R, Glaser R: ß-blockers and survival among Danish patients with malignant melanoma: a population-based cohort study. Cancer Epidemiol Biomarkers Prev 2011, 20:2273-2279.

10. Diaz ES, Karlan BY, Li AJ: Impact of beta blockers on epithelial ovarian cancer survival. Gynecol Oncol 2012, 127:375-378.

11. Danish Medicines Agency: Information on over-the-counter medicine and reimbursement criteria in Denmark. Available from URL: http:// laegemiddelstyrelsen.dk/en/ [accessed August 22, 2012].

12. Ehrenstein $V$, Antonsen S, Pedersen L: Existing data sources for clinical epidemiology: Aarhus University Prescription Database. Clin Epidemiol 2010, 2:273-279.

13. Pedersen CB: The Danish civil registration system. Scand J Public Health 2011, 39:22-25.

14. Gjerstorff ML: The Danish Cancer Registry. Scand J Public Health 2011 39:42-45.

15. Suissa S: Immortal time bias in pharmaco-epidemiology. Am J Epidemiol 2008, 167:492-499.

16. Lynge E, Sandegaard JL, Rebolj M: The Danish National Patient Register. Scand J Public Health 2011, 39:30-33. 
17. Charlson ME, Pompei $P$, Ales KL, Mackenzie CR: A new method of classifying prognostic comorbidity in longitudinal studies: development and validation. J Chronic Dis 1987, 40:373-383.

18. Needham DM, Scales DC, Laupacis A, Pronovost PJ: A systematic review of the Charlson comorbidity index using Canadian administrative databases: a perspective on risk adjustment in critical care research. J Crit Care 2005, 20:12-19.

19. Thygesen SK, Christiansen CF, Christensen S, Lash TL, Sorensen HT: The predictive value of ICD-10 diagnostic coding used to assess Charlson comorbidity index conditions in the population-based Danish National Registry of Patients. BMC Med Res Methodol 2011, 11:83.

20. Birim O, Kappetein AP, Bogers AJ: Charlson comorbidity index as a predictor of long-term outcome after surgery for nonsmall cell lung cancer. Eur J Cardiothorac Surg 2005, 28:759-762.

21. Singh B, Bhaya M, Stern J, Roland JT, Zimbler M, Rosenfeld RM, Har-El G, Lucente FE: Validation of the Charlson comorbidity index in patients with head and neck cancer: a multi-institutional study. Laryngoscope 1997, 107:1469-1475,

22. Glaser R, Zhang HY, Yao KT, Zhu HC, Wang FX, Li GY, Wen DS, Li YP: Two epithelial tumor cell lines (HNE-1 and HONE-1) latently infected with Epstein-Barr virus that were derived from nasopharyngeal carcinomas. Proc Natl Acad Sci USA 1989, 86:9524-9528.

23. Yang EV, Donovan EL, Benson DM, Glaser R: VEGF is differentially regulated in multiple myeloma-derived cell lines by norepinephrine. Brain Behav Immun 2008, 22:318-323.

24. Yang EV, Kim SJ, Donovan EL, Chen M, Gross AC, Webster Marketon JI, Barsky SH, Glaser R: Norepinephrine upregulates VEGF, IL-8, and IL-6 expression in human melanoma tumor cell lines: implications for stressrelated enhancement of tumor progression. Brain Behav Immun 2009, 23:267-275.

25. Yang EV, Sood AK, Chen M, Li Y, Eubank TD, Marsh CB, Jewell S, Flavahan $N A$, Morrison $C$, Yeh PE, et al: Norepinephrine up-regulates the expression of vascular endothelial growth factor, matrix metalloproteinase (MMP)-2, and MMP-9 in nasopharyngeal carcinoma tumor cells. Cancer Res 2006, 66:10357-10364.

26. Bangalore S, Kumar S, Kjeldsen SE, Makani H, Grossman E, Wetterslev J, Gupta AK, Sever PS, Gluud C, Messerli FH: Antihypertensive drugs and risk of cancer: network meta-analyses and trial sequential analyses of 324,168 participants from randomised trials. Lancet Oncol 2011, 12:65-82.

27. Storm HH: Completeness of cancer registration in Denmark 1943-1966 and efficacy of record linkage procedures. Int J Epidemiol 1988, 17:44-49.

28. Storm HH, Michelsen EV, Clemmensen IH, Pihl J: The Danish Cancer Registry - history, content, quality and use. Dan Med Bull 1997, 44:535-539.

doi:10.1186/1471-2407-13-85

Cite this article as: Johannesdottir et al: Use of B-blockers and mortality following ovarian cancer diagnosis: a population-based cohort study. BMC Cancer 2013 13:85.

\section{Submit your next manuscript to BioMed Central and take full advantage of:}

- Convenient online submission

- Thorough peer review

- No space constraints or color figure charges

- Immediate publication on acceptance

- Inclusion in PubMed, CAS, Scopus and Google Scholar

- Research which is freely available for redistribution 